\title{
An Efficient Solution Method for 0-1 Random Fuzzy Programming Problems Considering the Relaxation Problems
}

\author{
T. Hasuike ${ }^{*}$,, H. Ishii ${ }^{\mathrm{a}}$ and H. Katagiri ${ }^{\mathrm{b}}$ \\ ${ }^{a}$ Department of Information and Physical Sciences, Graduate School of Information Science and Technology, Osaka \\ Univeristy, Suita, Osaka, Japan \\ ${ }^{b}$ Department of Artificial Complex Systems Engineering, Graduate School of Engineering, Hiroshima University, \\ Higashi-Hiroshima, Hiroshima, Japan
}

\begin{abstract}
This paper considers a general 0-1 random fuzzy programming problem including some previous 0-1 stochastic and fuzzy programming problems. The proposal problem is not a well-defined problem due to including random fuzzy variables. Therefore, by introducing chance constraint and fuzzy goal for objective function and considering the maximization for the degrees of possibility that the objective function value satisfies the fuzzy goal, main problem is transformed into a deterministic equivalent problem. Furthermore, by using the assumption that each random variable is distributed according to a normal distribution, the problem is equivalently transformed into a basic $0-1$ programming problem, and the efficient strict solution method to find an optimal solution is constructed.
\end{abstract}

\section{INTRODUCTION}

0-1 programming problems are the most important mathematical models in practical managements and investments such as project selection problems, scheduling and facility location problems, and there are many previous academic and practical researches (recent academic studies; Balev [1], Freville [2], Jahanshahloo [3], Vasquez [4]).

In previous standard mathematical programming problems involving 0-1 programming problems, the coefficients of objective functions or constraints are assumed to be completely known and dealt with constant values. However, in practical systems, they should be treated as rather uncertain values than constant values due to probabilistic situations such as predictions of future profits or some machine troubles derived from historical data and ambiguous situations such as decision makers' substitutions. In order to consider such uncertainty, some uncertain models for mathematical programming problems have been introduced; stochastic programming problem (for example, Beale [5], Dantzig [6], Vajda [7]), fuzzy programming problem (for example, Inuiguchi [8], Sakawa [9], Zimmermann [10]) and the applications of fuzzy logics in various real cases (for example, Cheng [11] and Zhao [12]). Furthermore, Hasuike [13] has considered a 0-1 programming problem considering randomness and flexible goals for objective function and constraints. Katagiri [14] has considered a 0-1 programming problem involving both random and fuzzy conditions, i.e., fuzzy random 0-1 programming problem. Then, Katagiri [15] has considered a random fuzzy programming model based on possibilistic programming. Most recently, Huang

\footnotetext{
*Address correspondence to this author at the Graduate School of Information Science and Technology, Osaka University, 2-1 Suita, Osaka 565-0871, Japan; E-mail: thasuike@ist.osaka-u.ac.jp
}

[16] has proposed a project selection model including random fuzzy variables. However, there are few researches considering a general $0-1$ random fuzzy programming problem at this time. Therefore, this paper particularly considers the more general random fuzzy 0-1 programming problem maximizing the objective function involving random fuzzy variables, considering both the objectivity derived from statistical analysis of data and decision maker's subjectivity such as their institution derived from wide-ranging experiences, simultaneously. In this paper, we deal fuzzy numbers with L-R fuzzy numbers and random variables with continuous random distributions, particularly normal distributions.

On the other hand, generally speaking, solution methods of 0-1 programming problems mainly divides into two types; (a) strict solution methods such as dynamic programming and branch-bound method, (b) approximate solution methods such as genetic algorithm, heuristic methods, etc.. Furthermore, in mathematical programming, 0-1 programming problems considering randomness and fuzziness become more complicate models than the previous problems. Then, since these problem are not well-defined problems due to including both random variables and fuzzy numbers, it is almost impossible to solve them analytically. Therefore, in order to solve them directly, we need to set the target values for stochastic and fuzzy constraints and construct its efficient solution method. In this paper, we transform main problems into deterministic equivalent integer programming problems using chance constraints, possibility measure and fuzzy goals based on both stochastic and fuzzy programming approaches.

In Huang [16], a random fuzzy simulation based on approximate solution methods has been used. However, through the development of information technology and improvement of computers, we solve 0-1 programming problems more quickly using not only approximate solution 
methods but also strict solution methods even if they are little bit large scale problems. Therefore, in this paper, we propose the efficient strict solution method based on a mixed method with 0-1 relaxation problem and branch-bound method, and show the analytical efficiency comparing with previous strict solution methods.

\section{FORMULATION OF RANDOM FUZZY 0-1 PRO- GRAMMING PROBLEM}

A random fuzzy variable is one of the mathematical concepts dealing with randomness and fuzziness, simultaneously. In this paper, we deal with the random fuzzy variable based on the study of Liu [17].

\section{Definition 1 (Liu [17])}

A random fuzzy variable is a fuzzy set defined on a universal set of random variables.

Then, we formally introduce the following 0-1 programming problem:

Maximum $\overline{\boldsymbol{r}} \boldsymbol{x}$

subject to $\mathbf{A x} \leq \boldsymbol{b}, \boldsymbol{x} \in\{0,1\}^{n}$

where each notation is as follows:

A: $m \times n$ coefficient matrix

b: $m$-dimensional column vector

$\boldsymbol{x}$ : $n$-dimensional decision column vector (Decision variable)

The coefficient vector of objective function is $\overline{\tilde{\boldsymbol{r}}}=\left(\overline{\tilde{r}}_{1}, \overline{\tilde{r}}_{2}, \ldots, \overline{\tilde{r}}_{n}\right)$ and each $\overline{\tilde{r}}_{j}$ is a random fuzzy variable according to a normal distribution $N\left(\tilde{m}_{j}, \sigma_{j}^{2}\right)$ where $\tilde{m}_{j}$ is a mean value and $\sigma_{j}^{2}$ is a variance. Then, we represent the $i j$ th element of variance-covariance matrix as $\sigma_{i j}$. Furthermore, we assume that $\tilde{m}_{j}$ is a fuzzy variable characterized by the following membership function:

$\mu_{\tilde{m}_{j}}(\omega)=\left\{\begin{array}{cc}L\left(\frac{m_{j}-\omega}{\alpha_{j}}\right) & \left(m_{j}-\alpha_{j} \leq \omega \leq m_{j}\right) \\ R\left(\frac{\omega-m_{j}}{\beta_{j}}\right) & \left(m_{j}<\omega \leq m_{j}+\beta_{j}\right) \\ 0 & \left(\omega<m_{j}-\alpha_{j}, m_{j}+\beta_{j}<\omega\right)\end{array}\right.$

where $L(x)$ and $R(x)$ are nonincreasing reference function to satisfy $L(0)=R(0)=1, L(1)=R(1)=0$ and the parameters $\alpha_{j}$ and $\beta_{j}$ represent the spreads corresponding to the left and the right sides, respectively. Problem (1) is a random fuzzy 0-1 programming problem due to including random fuzzy variables. Then, its objective function $\overline{\tilde{Z}}=\overline{\tilde{\boldsymbol{r}} \boldsymbol{x}}$ is defined as a random fuzzy variable by the following membership function introducing a parameter $\bar{\gamma}_{j}$ and an universal set of normal random variable $\Gamma$ :

$\mu_{\overline{\tilde{Z}}}(\bar{u})=\sup _{\bar{\gamma}}\left\{\min _{1 \leq j \leq n} \mu_{\overline{\tilde{r}}_{j}}\left(\bar{\gamma}_{j}\right) \mid \bar{u}=\sum_{j=1}^{n} \bar{\gamma}_{j} x_{j}\right\}, \forall \bar{u} \in Y$ where $\bar{\gamma}=\left(\bar{\gamma}_{1}, \bar{\gamma}_{2}, \ldots, \bar{\gamma}_{n}\right), \mu_{\bar{r}_{j}}\left(\bar{\gamma}_{j}\right)$ is defined by

$\mu_{\overline{\tilde{r}}_{j}}\left(\bar{\gamma}_{j}\right)=\sup _{s_{j}}\left\{\mu_{\tilde{M}_{j}}\left(s_{j}\right) \mid \bar{\gamma}_{j} \sim N\left(s_{j}, \sigma_{j}^{2}\right)\right\}, \forall \bar{\gamma}_{j} \in \Gamma$

and $Y$ is defined by

$Y=\left\{\sum_{j=1}^{n} \bar{\gamma}_{j} x_{j} \mid \bar{\gamma}_{j} \in \Gamma, j=1,2, \ldots, n\right\}$

From these settings, we obtain

$$
\begin{aligned}
\mu_{\overline{\tilde{Z}}}(\bar{u}) & =\sup _{\bar{\gamma}}\left\{\min _{1 \leq j \leq n} \mu_{\overline{\tilde{r}}_{j}}\left(\bar{\gamma}_{j}\right) \mid \bar{u}=\sum_{j=1}^{n} \bar{\gamma}_{j} x_{j}\right\} \\
& =\sup _{\boldsymbol{s}}\left\{\min _{1 \leq j \leq n} \mu_{\tilde{M}_{j}}\left(s_{j}\right) \mid \bar{\gamma}_{j} \sim N\left(s_{j}, \sigma_{j}^{2}\right), \bar{u}=\sum_{j=1}^{n} \bar{\gamma}_{j} x_{j}\right\} \\
& =\sup _{s}\left\{\min _{1 \leq j \leq n} \mu_{\tilde{M}_{j}}\left(s_{j}\right) \mid \bar{u} \sim N\left(\sum_{j=1}^{n} s_{j} x_{j}, V(\boldsymbol{x})\right)\right\}
\end{aligned}
$$

where $V(x)=\sum_{i=1}^{n} \sum_{j=1}^{n} \sigma_{i j} x_{i} x_{j}$ Furthermore, we discuss the probability that the objective function value is greater than or equal to an aspiration level $f$. Then, we represent the probability as $\operatorname{Pr}\left\{\omega \mid \sum_{j=1}^{n} \overline{\tilde{r}}_{j} x_{j} \geq f\right\}$. Since $\sum_{j=1}^{n} \overline{\tilde{r}}_{j} x_{j}$ is represented with a random fuzzy variable, we express the probability $\operatorname{Pr}\left\{\omega \mid \sum_{j=1}^{n} \overline{\tilde{r}}_{j} x_{j} \geq f\right\}$ as a fuzzy set $\tilde{P}$ and defined the membership function of $\tilde{P}$ as follows:

$$
\begin{aligned}
\mu_{\tilde{P}}(p) & =\sup _{\bar{u}}\left\{\mu_{\tilde{\tilde{Z}}}(\bar{u}) \mid p=\operatorname{Pr}\{\omega \mid \bar{u}(\omega) \geq f\}\right\} \\
& =\sup _{\boldsymbol{s}} \min _{1 \leq j \leq n}\left\{\mu_{\tilde{M}_{j}}\left(s_{j}\right) \mid \begin{array}{l}
p=\operatorname{Pr}\{\omega \mid \bar{u}(\omega) \geq f\}, \\
\bar{u} \sim N\left(\sum_{j=1}^{n} s_{j} x_{j}, V(\boldsymbol{x})\right)
\end{array}\right\}
\end{aligned}
$$

where $s=\left(s_{1}, s_{2}, \ldots, s_{n}\right)$. Due to these randomness and fuzziness, problem (1) is not a well-defined problem, and so it is necessary to interpret the problem from some point of view and to transform the problem into the deterministic equivalent problem. In this paper, we consider the case where a decision maker prefers maximizing the degree of possibility for the probability that the value of objective function satisfies the fuzzy goal, based on previous research Katagiri [18] and Hasuike [19]. A fuzzy goal for the probability is characterized by the following membership function:

$\mu_{\tilde{G}_{P}}(p)=\left\{\begin{array}{cc}1 & p_{1}<p \\ g(p) & p_{0} \leq p \leq p_{1} \\ 0 & p<p_{0}\end{array}\right.$

where $g(y)$ is a monotonous increasing function. Then, using a concept of possibility measure, the degree of possibility that the objective function value satisfying a fuzzy goal $G$ is as follows:

$$
\prod_{P}(G)=\sup _{p} \min \left\{\mu_{\tilde{P}}(p), \mu_{\tilde{G}_{P}}(p)\right\}
$$


Consequently, problem (1) is transformed into the following problem:

Maximum $\prod_{P}(G)$

subject to $\mathbf{A x} \leq \boldsymbol{b}, \boldsymbol{x} \in\{0,1\}^{n}$

This problem is equivalently transformed into the following problem introducing a parameter $h$.

Maximum $h$

subject to $\prod_{P}(G) \geq h$,

$$
\mathbf{A x} \leq \boldsymbol{b}, \boldsymbol{x} \in\{0,1\}^{n}
$$

\section{DETREMINISTIC EQUIVALNET TRANSFORMA- TION OF THE PROPOSAL MODEL}

In problem (2), constraint $\prod_{P}(G) \geq h$ is transformed into the following inequality based on the result obtained by Katagiri [18] and Hasuike [19]:

$\prod_{P}(G) \geq h$

$\Leftrightarrow \sup _{p} \min \left\{\mu_{\tilde{P}}(p), \mu_{\tilde{G}_{P}}(p)\right\} \geq h$

$\Leftrightarrow \exists p: \mu_{\tilde{P}}(p) \geq h, \mu_{\tilde{G}_{P}}(p) \geq h$

$$
\begin{gathered}
\exists p: \sup _{s} \min _{1 \leq j \leq n}\left\{\mu_{\tilde{M}_{j}}\left(s_{j}\right) \mid \bar{u} \sim N\left(\sum_{j=1}^{n} s_{j} x_{j}, V(\boldsymbol{x})\right)\right\} \geq h, \\
\mu_{\tilde{G}_{p}}(p) \geq h
\end{gathered}
$$

$$
\exists p, \exists \boldsymbol{s}, \exists \bar{u}: \sup _{s} \min _{1 \leq j \leq n} \mu_{\tilde{M}_{j}}\left(s_{j}\right) \geq h,
$$$$
\Leftrightarrow \quad p=\operatorname{Pr}\{\omega \mid \bar{u}(\omega) \geq f\},
$$$$
\bar{u} \sim N\left(\sum_{j=1}^{n} s_{j} x_{j}, V(\boldsymbol{x})\right), \mu_{\tilde{G}_{P}}(p) \geq h
$$

$$
\exists p, \exists \boldsymbol{s}, \exists \bar{u}: \min _{1 \leq j \leq n} \mu_{\tilde{M}_{j}}\left(s_{j}\right) \geq h,
$$$$
\Leftrightarrow \quad p=\operatorname{Pr}\{\omega \mid \bar{u}(\omega) \geq f\},
$$$$
\bar{u} \sim N\left(\sum_{j=1}^{n} s_{j} x_{j}, V(\boldsymbol{x})\right), p \geq g^{-1}(h)
$$

$$
\exists p, \exists \boldsymbol{s}, \exists \bar{u}: \mu_{\tilde{M}_{j}}\left(s_{j}\right) \geq h,(j=1,2, \ldots, n)
$$$$
\Leftrightarrow \quad \bar{u} \sim N\left(\sum_{j=1}^{n} s_{j} x_{j}, V(\boldsymbol{x})\right)
$$

$$
\operatorname{Pr}\{\omega \mid \bar{u}(\omega) \geq f\} \geq g^{-1}(h)
$$

$$
\exists \bar{u}: \operatorname{Pr}\{\omega \mid \bar{u}(\omega) \geq f\} \geq g^{-1}(h),
$$$$
\Leftrightarrow \quad \bar{u} \sim N\left(\sum_{j=1}^{n}\left(m_{j}+R^{*}(h) \alpha_{j}\right) x_{j}, V(\boldsymbol{x})\right),
$$

where $R^{*}(x)$ is a pseudo inverse function of $R(x)$. From this inequality, problem (2) is equivalently transformed into the following problem:
Maximum $h$

subject to $\operatorname{Pr}\{\omega \mid \bar{u}(\omega) \geq f\} \geq g^{-1}(h)$,

$$
\begin{aligned}
& \bar{u} \sim N\left(\sum_{j=1}^{n}\left(m_{j}+R^{*}(h) \alpha_{j}\right) x_{j}, V(\boldsymbol{x})\right), \\
& \mathbf{A} \boldsymbol{x} \leq \boldsymbol{b}, \boldsymbol{x} \in\{0,1\}^{n}
\end{aligned}
$$

Furthermore, with respect to stochastic constraint $\operatorname{Pr}\{\omega \mid \bar{u}(\omega) \geq f\} \geq g^{-1}(h)$, by using the property of normal distribution, this constraint is equivalently transformed into the following form:

$\operatorname{Pr}\{\omega \mid \bar{u}(\omega) \geq f\} \geq g^{-1}(h)$

$$
\Leftrightarrow \frac{\sum_{j=1}^{n}\left(m_{j}+R^{*}(h) \alpha_{j}\right) x_{j}-f}{\sqrt{V(\boldsymbol{x})}} \geq K_{g^{-1}(h)}
$$

where $F(z)$ is the distribution function of the standard normal distribution and $K_{t}=F^{-1}(t)$. Furthermore, each decision variable $x_{j}$ satisfies $x_{j} \in\{0,1\}$, we obtain $x_{j}{ }^{2}=x_{j}$, and assume that each vairiance is independent, i.e.,

$\sigma_{i j}=\left\{\begin{array}{cc}\sigma_{j}^{2} & (i=j) \\ 0 & (i \neq j)\end{array}\right.$

Consequently, problem (3) is equivalently transformed into the following problem:

Maximum $h$

$$
\begin{gathered}
\text { subject to } \frac{\sum_{j=1}^{n}\left(m_{j}+R^{*}(h) \alpha_{j}\right) x_{j}-f}{\sqrt{\sum_{j=1}^{n} \sigma_{j}^{2} x_{j}}} \geq K_{g^{-1}(h)}, \\
\mathbf{A} \boldsymbol{x} \leq \boldsymbol{b}, \boldsymbol{x} \in\{0,1\}^{n}
\end{gathered}
$$

It should be noted here that problem (4) is a nonconvex integer programming problem and it is not solved by the linear programming techniques or convex programming techniques. However, since a decision variable $h$ is involved only in first constraint, we introduce the following subproblem involving a parameter $q$ :

$\operatorname{Maximum} \frac{\sum_{j=1}^{n}\left(m_{j}+R^{*}(q) \alpha_{j}\right) x_{j}-f}{\sqrt{\sum_{j=1}^{n} \sigma_{j}^{2} x_{j}}}$

subject to $\mathbf{A x} \leq \boldsymbol{b}, \boldsymbol{x} \in\{0,1\}^{n}$

In the case that we fix the parameter $q$, problem (5) is equivalent to a convex integer programming problem. Furthermore, let $\boldsymbol{x}(q)$ and $Z(q)$ be an optimal solution of problem (5) and its optimal value, respectively. Then, the following theorem is derived from previous study $[18,19]$.

\section{Theorem 1}

For $q$ satisfying $0<q<1, Z(q)$ is a strictly increasing function of $q$.

Furthermore, let $\hat{q}$ denote $q$ satisfying $Z(\hat{q})=g^{-1}(\hat{q})$ and the optimal solutions of main problem (4) be $\left(x^{*}, h^{*}\right)$. Then 
the relation between problems (4) and (5) is derived as follows derived from previous study [20].

\section{Theorem 2}

Suppose that $0<\hat{q}<1$ holds. Then $(x(\hat{q}), \hat{q})$ is equal to $\left(x^{*}, h^{*}\right)$.

From these theorems, by using bisection algorithm for parameter $q$ and comparing objective function $Z(q)$ with $g^{-}$ ${ }^{1}(q)$, we repeatedly solve problem (5) for each $q$ using branch-bound method, and finally obtain the optimal solution. This solution method is assured that its calculation times are infinite. However, it is not efficient due to increasing computational times voluminously with the increase of parameters and decision variables. Therefore, we need to construct the more efficient solution method.

\section{CONSTRCUTION OF THE EFFICIENT STRICT SO- LUTION METHOD}

In order to construct the efficient strict solution method for problem (4), first of all, we introduce the following 0-1 relaxation problem of problem (4):

Maximum $h$

$$
\begin{gathered}
\text { subject to } \frac{\sum_{j=1}^{n}\left(m_{j}+R^{*}(h) \alpha_{j}\right) x_{j}-f}{\sqrt{\sum_{j=1}^{n} \sigma_{j}^{2} x_{j}}} \geq K_{g^{-1}(h)}, \\
\mathbf{A} \boldsymbol{x} \leq \boldsymbol{b}, 0 \leq x_{j} \leq 1, j=1,2, \ldots, n
\end{gathered}
$$

In a way similar to problem (4), this problem is also a nonconvex programming problem and it is not solved by the linear programming techniques or convex programming techniques. Subsequently, we introduce the following subproblem:

$$
\operatorname{Maximum} \frac{\sum_{j=1}^{n}\left(m_{j}+R^{*}(q) \alpha_{j}\right) x_{j}-f}{\sqrt{\sum_{j=1}^{n} \sigma_{j}^{2} x_{j}}}
$$

subject to $\mathbf{A} \boldsymbol{x} \leq \boldsymbol{b}, 0 \leq x_{j} \leq 1, j=1,2, \ldots, n$

In this paper, it is assumed that there exists a feasible solution satisfying $\sum_{j=1}^{n}\left(m_{j}+R^{*}(q) \alpha_{j}\right) x_{j}>f$. This means that the probability that total future profit is more than target value $f$ is greater than $1 / 2$. Furthermore, problem (7) is equivalent to the following problem:

$$
\text { Minimum } \frac{\sqrt{\sum_{j=1}^{n} \sigma_{j}^{2} x_{j}}}{\sum_{j=1}^{n}\left(m_{j}+R^{*}(q) \alpha_{j}\right) x_{j}-f}
$$

subject to $\mathbf{A} \boldsymbol{x} \leq \boldsymbol{b}, 0 \leq x_{j} \leq 1, j=1,2, \ldots, n$

In the case we fix the parameter $q$, since problem (8) is a nonlinear fractional programming problem due to including a square root term $\sqrt{\sum_{j=1}^{n} \sigma_{j}^{2} x_{j}}$ in the objective function, it is difficult to solve this original problem directly. Therefore, we introduce the following parameters;

$$
t=\frac{1}{\sum_{j=1}^{n}\left(m_{j}+R^{*}(q) \alpha_{j}\right) x_{j}-f}, \boldsymbol{y}=\boldsymbol{t} \boldsymbol{x}
$$

and we do the transformation into the following deterministic equivalent problem:

$$
\begin{aligned}
& \text { Minimum } \sqrt{\sum_{j=1}^{n} \sigma_{j}^{2} y_{j}} \\
& \text { subject to } \sum_{j=1}^{n}\left(m_{j}+R^{*}(q) \alpha_{j}\right) y_{j}-f t=1, \\
& \mathbf{A} \boldsymbol{y} \leq \boldsymbol{b t}, 0 \leq y_{j} \leq t, j=1,2, \ldots, n
\end{aligned}
$$

Since objective function $\sqrt{\sum_{j=1}^{n} \sigma_{j}^{2} y_{j}}$ is a monotonous increasing function, this problem is equivalently transformed into the following problem:

$$
\begin{array}{ll}
\text { Minimum } & \sum_{j=1}^{n} \sigma_{j}^{2} y_{j} \\
\text { subject to } & \sum_{j=1}^{n}\left(m_{j}+R^{*}(q) \alpha_{j}\right) y_{j}-f t=1, \\
& \mathbf{A} \boldsymbol{y} \leq \boldsymbol{b} t, 0 \leq y_{j} \leq t, j=1,2, \ldots, n
\end{array}
$$

Problem (9) is a linear programming problem in the case that $q$ is fixed, and so we efficiently obtain the optimal solution using linear programming approaches and bisection algorithm for parameter $q$.

Furthermore, let the optimal value of parameter $h$ in problem (4) be $h^{*}$. Then, the following lemmas hold:

\section{Lemma 1}

With respect to problem (4), there exists ranges $\left[h_{k}, h_{k+1}\right]$ $(k=1,2, \ldots)$ that the optimal solution of problem (4) is unique for any $h$ including in $\left[h_{k}, h_{k+1}\right]$.

\section{Proof}

From the continuity of parameter $h$ and discreteness of decision variable $\boldsymbol{x}^{*}$, this lemma clearly holds.

\section{Lemma 2}

Let the optimal value of problem (6) be $\bar{h}$, the optimal solution of problem (4) be $x^{*}$ and the optimal value be $h^{*}$. Then in the case that we set a range $\left[h_{L}, h_{U}\right]$ satisfying $\bar{h} \in$ $\left[h_{L}, h_{U}\right], h^{*} \in\left[h_{L}, h_{U}\right]$ holds.

\section{Proof}

We consider the case that $h^{*} \notin\left[h_{L}, h_{U}\right]$ and $h^{*} \in\left[h_{L}^{\prime}, h_{U}^{\prime}\right]$. If $h_{U}^{\prime}<h_{L}$, there exists the optimal solution $x^{\prime}$ and the optimal solution $h^{\prime}$ satisfying $h^{\prime} \in\left[h_{L}, h_{U}\right]$. This contradicts the optimality of parameter $h^{*}$. In a way similar to $h_{U}^{\prime}<h_{L}$, if $h_{L}^{\prime}>h_{U}$, we obviously find that $h_{L}^{\prime}>h^{*}$. This means that the optimal value of discrete problem is larger than that of continuous problem, and contradicts the optimality of parameter $\bar{h}>h^{*}$. Consequently, this lemma holds. 
From these lemmas, the following theorem to the relation between problems (4) and (6) holds:

\section{Theorem 3}

Let the optimal solution of problem (6) be $\boldsymbol{x}^{*}(\bar{h})$ and the optimal value of parameter $h$ be $\bar{h}$. Then, the optimal solution of the following problem;

Maximum $\frac{\sum_{j=1}^{n}\left(m_{j}+R^{*}(\bar{h}) \alpha_{j}\right) x_{j}-f}{\sqrt{\sum_{j=1}^{n} \sigma_{j}^{2} x_{j}}}$

subject to $\mathbf{A x} \leq \boldsymbol{b}, \boldsymbol{x} \in\{0,1\}^{n}$

is equivalent to that of problem (4).

Consequently, in the case that we solve 0-1 relaxation problem (6) and obtain its optimal solution $h^{*}$, we obtain an optimal solution more efficiently than previous parametric approaches due to not using branch-bound method every value of parameter $h$ repeatedly. However, since the objective function of problem (10) is a nonlinear function, it is not easy to deal with several efficient solution methods for integer programming approaches. Therefore, in order to have the more general versatility for our proposed model, we consider the other deterministic equivalent transformations for main problem.

First, we equivalently transform main problem (4) into the following problem;

Maximum $h$

subject to $\sum_{j=1}^{n}\left(m_{j}+R^{*}(h) \alpha_{j}\right) x_{j}-K_{g^{-1}(h)} \sqrt{\sum_{j=1}^{n} \sigma_{j}^{2} x_{j}} \geq f$,

$$
\mathbf{A x} \leq \boldsymbol{b}, \boldsymbol{x} \in\{0,1\}^{n}
$$

and introduce this $0-1$ relaxation problem as follows:

Maximum $h$

$\begin{array}{ll}\text { subject to } & \sum_{j=1}^{n}\left(m_{j}+R^{*}(h) \alpha_{j}\right) x_{j}-K_{g^{-1}(h)} \sqrt{\sum_{j=1}^{n} \sigma_{j}^{2} x_{j}} \geq f, \\ & \mathbf{A} \boldsymbol{x} \leq \boldsymbol{b}, 0 \leq x_{j} \leq 1, j=1,2, \ldots, n\end{array}$

This problem is a nonlinear programming problem. However, this problem is much similar to problem (6). Therefore, in order to solve problem (11) analytically, we introduce the following subproblem in a way similar to the transformation from problem (6) into problem (7):

Maximum $\sum_{j=1}^{n}\left(m_{j}+R^{*}(h) \alpha_{j}\right) x_{j}-K_{g^{-1}(h)} \sqrt{\sum_{j=1}^{n} \sigma_{j}^{2} x_{j}}$

subject to $\mathbf{A} \boldsymbol{x} \leq \boldsymbol{b}, 0 \leq x_{j} \leq 1, j=1,2, \ldots, n$

Then, we consider the following auxiliary problem:

Maximum $\gamma \sum_{j=1}^{n}\left(m_{j}+R^{*}(h) \alpha_{j}\right) x_{j}-K_{g^{-1}(h)}\left(\sum_{j=1}^{n} \sigma_{j}^{2} x_{j}\right)$

subject to $\mathbf{A} \boldsymbol{x} \leq \boldsymbol{b}, 0 \leq x_{j} \leq 1, j=1,2, \ldots, n$

In the case that we fix parameter $h$, with respect to the relation between problems (13) and (14), the following theorem holds based on the previous research of Ishii [21].

\section{Theorem 4}

Let the optimal solution of problem (13) be $\boldsymbol{x}(h)$. Then, in the case $\gamma=2 \sqrt{\sum_{j=1}^{n} \sigma_{j}^{2} x_{j}^{*}}$, the optimal solution of problem (14) is equal to $\boldsymbol{x}(h)$.

From Theorem 4, in the case that parameter $h$ is fixed, we obtain the optimal solution $\boldsymbol{x}(h)$. Furthermore, we consider the following problem to deal with optimal value $\bar{h}$ of problem (6):

$$
\text { Maximum } \gamma \sum_{j=1}^{n}\left(m_{j}+R^{*}(\bar{h}) \alpha_{j}\right) x_{j}-K_{g^{-1}(\bar{h})}\left(\sum_{j=1}^{n} \sigma_{j}^{2} x_{j}\right)
$$$$
\text { subject to } \mathbf{A} \boldsymbol{x} \leq \boldsymbol{b}, 0 \leq x_{j} \leq 1, j=1,2, \ldots, n
$$

Let this optimal solution be $\boldsymbol{x}\left(h^{*}\right)$. Subsequently, the following lemma with respect to each optimal solution for problems (4) and (11) holds.

\section{Lemma 3}

The optimal solution of problem (11) is equal to that of problem (4).

\section{Proof}

Since each problem is the deterministic equivalent problem for main problem (3), this lemma obviously holds.

Therefore, we obtain $\boldsymbol{x}(\bar{h})=\boldsymbol{x}^{*}$. Then, the following theorem holds extending previous research of Hasuike.

\section{Theorem 5}

With respect to $\gamma^{*}=2 \sqrt{\sum_{j=1}^{n} \sigma_{j}^{2} x_{j}^{*}}$, the optimal solution of the following problem;

$$
\begin{aligned}
& \text { Maximum } \gamma^{*} \sum_{j=1}^{n}\left(m_{j}+R^{*}(\bar{h}) \alpha_{j}\right) x_{j}-K_{g^{-1}(\bar{h})}\left(\sum_{j=1}^{n} \sigma_{j}^{2} x_{j}\right) \\
& \text { subject to } \mathbf{A} \boldsymbol{x} \leq \boldsymbol{b}, \boldsymbol{x} \in\{0,1\}^{n}
\end{aligned}
$$

is equal to that of problem (3).

From Theorem 5, we finally solve this linear $0-1$ programming problem. It is more efficient to obtain its optimal solution of problem (11) using some efficient solution methods for integer programming approaches than that of problem (4). Furthermore, in the case using branch-bound method, we find that upper limited value for main problem becomes

$$
\sum_{j=1}^{n}\left(m_{j}+R^{*}(\bar{h}) \alpha_{j}\right) x_{j}(\bar{h})-K_{g^{-1}(\bar{h})} \sqrt{\sum_{j=1}^{n} \sigma_{j}^{2} x_{j}(\bar{h})}
$$

substituting optimal solution $x(\bar{h})$ and optimal value $\bar{h}$ of problem (6) and lower limited value becomes $f$. Therefore, by using these values in branch-bound method efficiently, we obtain the optimal solution of main problem more easily and rapidly. Consequently, we construct the following solution method. 


\section{Solution Method}

STEP 1: Elicit the membership function of a fuzzy goal for with respect to the probability and set each parameter.

STEP 2: Solve 0-1 relaxation problem (6), and find the optimal solution $\boldsymbol{x}^{*}$ and optimal value $\bar{h}$.

STEP 3: Solve 0-1 programming problem (16) by using integer programming approaches such as branch-bound method.

\section{CONCLUSIONS}

In this paper, we have proposed a general 0-1 random fuzzy programming problem considering both random and fuzzy conditions. Since our proposed model has been a nonlinear $0-1$ programming problem by introducing the chance constraint and doing the transformation into the deterministic equivalent problems, we have constructed the efficient strict solution method by dealing with some 0-1 relaxation problems. Consequently, we have found that the number of using branch-bound method in our proposed method is much less than that in previous parametric solution methods.

This solution method may be applicable to the general integer programming problems because our proposal model includes some previous models not considering randomness or fuzziness. However, in the case there are many decision variables and parameters, it takes much computational time to solve this 0-1 random fuzzy programming problem even if we use this solution method due to the nonpolynomial time algorithm to branch-bound method. Therefore, as future studies, we need to construct its efficient solution method using not only strict solution method such as branch-bound method but also approximation methods such as genetic algorithm and heuristic approaches. Futhermore, we consider the multidimensional $0-1$ and integer random fuzzy programming problems.

\section{REFERENCES}

[1] Balev S, Yanev N, Fréville A, Andonov R. A dynamic programming based reduction procedure for the multidimensional $0-1$ knapsack problem. Eur J Oper Res 2008; 186: 63-76.
[2] Freville A. The multidimensional 0-1 knapsack problem: An overview. Eur J Oper Res 2004; 155: 1-21.

[3] Jahanshahloo GR, Hosseinzadeh F, Shoja H, Tohidi G. A method for generating all efficient solutions of 0-1 multi-objective linear programming problem. Appl Math Comput 2005; 169: 874-886.

[4] Vasquez M, Vimont V. Improved results on the 0-1 multidimensional knapsack problem. Eur J Oper Res 2005; 165: 70-81.

[5] Beale EML. On optimizing a convex function subject to linear inequalities. J Royal Stat Soc 1955; 17: 173-184.

[6] Dantzig GB. Linear programming under uncertainty. Manag Sci 1955; 1: 197-206.

[7] Vajda S. Probabilistic Programming. Academic Press 1982.

[8] Inuiguchi M, Ichihashi H, Tanaka H. Fuzzy programming: A survey of recent developments. In: Slowinski R, Teghem J, Eds. Stochastic versus Fuzzy Approaches to Multiobjective mathematical programming under Uncertainty: Kluwer Academics 1990; pp. 4568.

[9] Sakawa M. Fuzzy Sets and Interactive Optimization. Plenum 1993.

[10] Zimmermann HJ. Fuzzy programming and linear programming with several objective functions. Fuzzy Sets Syst 1978; 1: 45-55.

[11] Cheng CT, Chau KW. Three-person multi-objective conflict decision in reservoir flood control. Eur J Oper Res 2002; 142: 625-631.

[12] Zhao MY, Cheng CT, Chau KW, Li G. Multiple crteira data envelopment analysis for full ranking units associated to environment impact assessment. Int J Environ Pollut 2006; 28: 448-464.

[13] Hasuike T, Ishii H. A 0-1 knapsack problem considering randomness of future returns and flexible goals of available budget and total return. Sci Math Japonicae e-2008; 273-283.

[14] Katagiri H, Sakawa M, Kato K, Nishizaki I. A Fuzzy Random Multiobjective 0-1 Programming Based on the Expectation Optimization Model Using Possibility and Necessity Measure. Math Comput Model 2004; 40: 411-421.

[15] Katagiri H, Hasuike T, Ishii $\mathrm{H}$. A random fuzzy programming model based on possibilistic programming. Proceedings of IEEE International Conference on Systems, Man, and Cybernetic 2008; in press.

[16] Huang X. Optimal project selection with random fuzzy parameters. Int J Product Economics 2007; 106: 513-522.

[17] Liu B. Theory and Practice of Uncertain Programming. Physica Verlag 2002.

[18] Katagiri H, Ishii H, Sakawa M. Linear programming problems with random fuzzy variable coefficients. Proceedings of 5th CzechJapan Seminar on Data Analysis and Decision Making under Uncertainty $2002 ; 55-58$.

[19] Hasuike T, Katagiri H, Ishii H. Probability Maximization Model of 0-1 Knapsack Problem with Random Fuzzy Variables. Proc FUZZIEEE 2008; 548-54.

[20] Katagiri H, Ishii H, Sakawa M. On fuzzy random linear knapsack problems. Central Eur J Oper Res 2004; 12: 59-70.

[21] Ishii H, Shiode S, Nishida T, Namasuya Y. Stochastic spanning tree problem. Discrete Appl Math 1981; 3: 263-373. 